\title{
Two-Sided Search and Temporary Employment
}

\author{
Dimitri Paolini ${ }^{x}$ \\ IRES, Université Catholique de Louvain \\ 3 Place Montesquieu; B1348 Louvain La Neuve, Belgium \\ paolini@ires.ud.ac.be
}

May 2000

\begin{abstract}
A bstract
Theobjective of this paper is to model explicitly the possibility to form temporary matching in a model of two-sided search. The agents (workers and employers) dixer in their human quality endowment. In a search equilibrium agents form subintervals and are only matched to agents within their class. The introduction of a temporary market can have a positive impact on unemployment, but it may have a negative impact on low-skilled agents' utility. When a delay cost is introduced, this negative impact can be reduced especially if an education policy is implemented to decrease the heterogeneity of the human capital.

K eywords: Two-Sided Search, Matching, Temporary Employment.

J EL Classi..cation: C78, J 41, J 64.
\end{abstract}

\footnotetext{
"I would liketo thank L. Artige, A. Gautier, R. Nicolini, G. Parodi, S. Peralta, and especially F. Bloch for his very helpful comments and discussions. I am also grateful to seminar participants at DMQTE and YEC Annual Meeting Oxford. Remaining errors are mine. The ..nancial support of the PAI program $\mathrm{P} 4 / 01$ is gratefully acknowledged.
} 


\section{Introduction}

The share of temporary work in total employment has been increasing in Europe in recent years. As Cahuc and Postel-Vinay (1999) point out, at the end of the seventies, labor market regulations restricted temporary jobs were destined to speci..c tasks, characterized by large variations in productivity. Those regulations have changed somewhat since the eighties, and it is now possible in a number of European countries to hire workers on a temporary basis even for jobs which are not subject to such variations in productivity.

While in 1983 only 4\% of the employees in the EC held temporary jobs, in 1991 this ..gure had rose to10\% did (see Table 1).

\begin{tabular}{ll|ll}
\hline Country & Share (\%) & Country & Share (\%) \\
\hline Belgium & 5.1 & Germany & 10.5 \\
UK & 5.4 & France & 11 \\
Italy & 7.5 & Portugal & 11 \\
Ireland & 9 & Denmark & 11.5 \\
Netherlands & 10 & Finland & 13 \\
Greece & 10.5 & Spain & 33 \\
\hline
\end{tabular}

Table 1: Share of temporary jobs in total employment (source: OECD (1996), quoted in Cahuc and Postel-Vinay (1999))

Using a panel of Spanish .r.rms, Bentolila and Dolado (1994) and Bentolila and SaintPaul (1992) show that the introduction of ..xed-duration contracts is equivalent to a reduction in ..ring cost and that its impact on unemployment is ambiguous.

Wasmer (1999), Cahuc and Postel-Vinay (1999), in a theoretical model, have introduced temporary job in matching models. The former, in a mode with exogenous job destruction, shows that in the periods of low growth the .r.rms are more willing to make use of temporary contracts, which is favorable to employment. The latter, in a model with endogenous job destruction, show that the combination of temporary jobs and ..ring restriction may be both inet cient in terms of aggregate welfare and an inade quate weapon to ..ght unemployment. This result comes from the fact that the share of temporary jobs transformed into permanent jobs is decreasing in the level of the ..ring cost.

The objective of this paper is to model explicitly the possibility to form temporary matching in a model of two-sided search. We use this model to study the impact of labor force heterogeneity, education, and the eq ciency on the search process on both the unemployment and the formation of the matching equilibria. We impose conditions that guarantee the existence of a Steady State equilibrium and then characterize it.

In labor, marriage, and related markets, the central problem is the creation of cooperating coalitions composed of two or more agents of dixerent types, eg., worker and 
employer, man and woman, etc. In the labor market case, a cooperating coalition is a producing unit composed of a job-worker match. The job-worker match is formed when an unemployed worker and a vacancy meet, and they agree to sign a contract.

There is a growing literature examining non-cooperative matching models in which populations of heterogeneous agents are matched. The matching literature focuses on the ways in which the search strategies of agents infuence outcomes for all agents. Recent contributions to this literature are Bloch and Ryder (2000), MacNamara and Collins (1990), Morgan (1994), Burdett and Coles (1997) and Smith (1995).

In the former three articles the matching problem is simpli.eed by assuming that any pair who leaves the market is immediately replaced by clones. In the Burdett and Coles' mode, on the contrary, there is an exogenous infow of new singles into the market. Clearly, the Steady State Equilibrium requires that the distribution of types of those who exit equals the infow distribution ${ }^{1}$. This extension is important because not only it best capture the characteristics of the labor market but, moreover it allows the matching decisions to inłuence the Steady State distributions of types in the market. This extension is important because not only it captures the characteristics of the labor market in the most appropriate manner, but also it allows the matching decisions to inłuence the Steady State distributions of types in the market.

Burdett and Coles provide a proof for the case where multiple class equilibria arises in a marriage market with two-sided heterogeneity and with exogenous in $\ddagger$ ows of new agents, even when there are constant returns to matching. The equilibrium requires that all agents use utility-maximizing strategies, given the behavior of other agents, where a strategy is a list of people to whom a particular single will propose a matching. In such a framework, the optimal strategy for an agent $\mathrm{i}$ is to accept all the partners whose human capital is above a critical level and to reject all the agents whose human capital is below this reservation level. In their ..r.st proposition Burdett and Coles show that in equilibrium the participants partition themselves into $n$ distinct classes and that $a$ woman (employer) in class $n$ will only propose to men (workers) who are in the same class or higher $n$, and will al ways reject a man from a lower class. Men do the same. In equilibrium only the men and women from the same class marry.

We consider a discrete version of Burdett and Coles (1997) and we introduce the possibility of forming a temporary match through a centralized temporary agency. The temporary agency will match the agents at random because we suppose that it can not distinguish the agents' quality.

Employers and workers have two options: they can look for a partner in the search market or look for a temporary match through a specialized agency (temporary agency).

Formally, both workers and employers are characterized by an index of human quality. We analyze the simple case of a binomial distribution of human capital.

\footnotetext{
${ }^{1}$ Smith (1995) assumes a nonsteady state matching dynamics through the time.
} 
In the ..rst part of the paper, we introducethe basic model of two-sided search, where agents meet randomly every period and simultaneously decide at each meeting whether to sign a contract. Search costs are captured by a discount factor, which captures the agents' impatience to form a matching. We will show that in the case of a binomial distribution of types there are two possible equilibria, and that these equilibria depend on the degree of human capital heterogeneity, the search ec ciency, and the proportion of educated.

In the second part of the paper, we introduce the possibility to form temporary matching, through a centralized temporary agency. Weassumethat if theagents consider the agent they met in the search market not acceptable or if they do not meet anybody, they can either decide to wait until the next matching or to match temporarily, in the temporary market. We impose that the temporary contract lasts two periods. First of all, we make the hypothesis that the agents can enter and exit the temporary market without delay. Then, we analyze the case where a delay cost to enter this market is introduced.

This paper shows that the introduction of a temporary market has the exect of reducing the key importance of the search ec ciency and the proportion of educated in determining of the equilibrium. Moreover, if on the one hand it has a positive impact on unemployment, on the other hand it implies less opportunities for less skilled agents. These can just try to stay as long as possible in the temporary market in the hope of forming a temporary match with a high-skilled agent. The best way to improve lowskilled agents' welfare is to give all of them a higher quality education: as human capital heterogeneity decreases, skilled-agents will be more willing to accept less-educated agents as partners. This policy should always be accompanied by a temporary market regulation to avoid that the improvement of quality education loses its positive exect.

The rest of the paper is organized as follows: in the next section we present the model. In section 3 we look at a model without temporary market. In section 4 we introduce the temporary market. In section 5 we make some comparative statics and Section 6 provides some condusions.

\section{The Model}

\subsection{Workers and Employers}

The mode is a discrete time, two-sided search with heterogeneous agents.

The economy is composed of two types of agents: high-skill $(\mathrm{H})$ and low-skill $(\mathrm{L})$. $H$ and $L$ agents are endowed with human capital $x_{h}=1+\varnothing$ and $x_{l}=1$ i $\varnothing$ where $\varnothing$ measures the degree of human capital heterogeneity and subscripts denote the type of the agent. 
All employers (workers) have identical preferences over the set of the workers (employers). The utility obtained corresponds to the human capital of the worker (employer) matched.

$$
\begin{aligned}
& u_{w}\left(x_{I}\right)=u_{e}\left(x_{I}\right)=x_{l} \\
& u_{w}\left(x_{h}\right)=u_{e}\left(x_{h}\right)=x_{h}
\end{aligned}
$$

\subsection{M arket}

Let us suppose that a large and equal number of workers and employers is looking for a partner. $\mathrm{N}(\mathrm{t})$ denotes the number of workers (employers) on the market at period $\mathrm{t}$.

There are two markets: a search market and a temporary market. The workers and the employers have two options: they can look for a partner in the search market or look for a temporary match through a specialized agency (temporary agency). Thetemporary agency matches the agents at random because we suppose that it cannot distinguish their qualities. Time is discrete and runs as $t=0 ; 1 ; \ldots .: ;,+1$. At any date $t$, the worker and the employer are matched according to a simple random matching technology. Arrival follows a Poisson process, where ${ }^{\circledR}$ is the arrival rate of a worker (employer) faced by an employer (worker). As ${ }^{\circledR}$ is assumed to be independent of the number of participants to the search market, the matching function exhibits constant returns.

Let ${ }^{-}>0$ denote the probability that any individual dies in an interval of time. To simplify the turnover dynamics, assume that an agent never returns to this market once a match has been formed, including the case when the partner has died.

Let, $w=, e=$, denote the number of new workers and new employers who enter the market in any time interval. We further assume that the distribution of types who fow into the market in any period is the same for workers and for employers; i.e, $F_{w}(\Phi)=F_{e}(\Phi)=F(\emptyset$. In particular, we suppose that there is a constant proportion of $\mathrm{H}$ individuals fowing into the market at any period and let this proportion be denoted ${ }^{\prime}{ }_{w}{ }^{\prime}{ }_{e}={ }^{\prime}$. Further suppose that the proportion of singles that have type $\mathrm{H}$ at time $\mathrm{t}$ is $1 / \mathrm{At})$.

All agents have a common intertemporal discount factor denoted $\pm>0$, and both worker and employer obtain zero utility when single. The discount factor captures the search cost.

\subsection{Timing}

At each meeting in the search market the worker and employer can observe the type of the other one. If both propose to sign a contract, they form a match and leave the market. On the other hand, if at least one of the two agents does not accept to sign the 


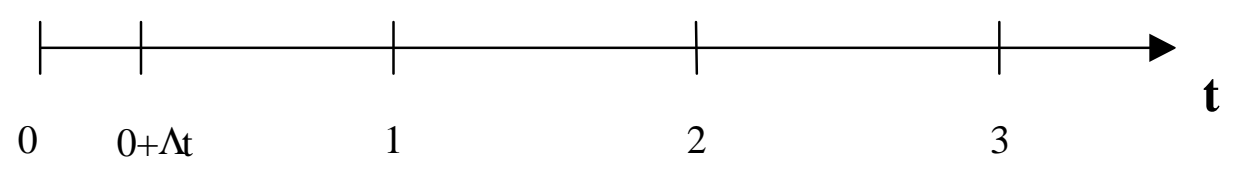

Figure 1:

contract, they remain in the market. They can then decide to ...nd a temporary matching through the temporary agency or wait to have a new possibility of matching in the next period.

Our analysis is based on three models: the basic model without temporary employment (model I), a model with temporary employment and no delay cost in thetemporary market (mode II), and one with temporary employment and with delay cost in thetemporary market (model III).

All thre models have the following time sequence:

M odel I In thenext section we will analyze the simple case without temporary agency.

In $\mathrm{t}=0$ the agent has a probability to meet somebody; if he meets somebody and both ...nd the matching acceptable, they leave the market for life The agents start enjoying the utility when they sign the contract. On the contrary, if a worker (employer) ..nds the matching unacceptable or if he does not match with anybody (which happens with probability $\left(1_{i} \otimes\right)$ ), he can try to form a new match in $t=1$.

M odel II In the third section we give the agent the possibility to form a temporary matching through the temporary agency. We suppose that after the temporary matching the agent can come back directly in the market without delay.

In $\mathrm{t}=0$ if the agent does not match with anybody, he can either decide to wait until $\mathrm{t}=1$ or to go directly to the temporary agency, in $\mathrm{t}=0+\alpha \mathrm{t}$, and look for a temporary matching. If an agent signs a contract for temporary employment, he remains committed for two periods, so he goes back to the market only at $t=2$.

We impose the two period duration to the temporary contract because, were it to last only for 1 period, everyone would be better ox by trying the temporary market after a failure in the search market.

This can be interpreted as an opportunity cost of entering the temporary market.

M odel III In the last model we will introduce a cost in the temporary market. After forming a temporary matching the agent has to wait for a period (delay cost) before 
being able to search for a partner again (he can only come back at $t=3$ ).

\section{The Search Equilibrium}

Before introducing the possibility to form a temporary matching, we present the basic model without temporary agency (TA). The model is a discrete time two-sided search in the fashion of Burdett and Coles (1997).

In the case of a binomial distribution of types, without temporary agency, there are two possible types of equilibria, characterized by whether high-skill types are willing to work with low-skill types. We will refer to the ..rst equilibrium as a Single Class Equilibrium (SCE), and to the second one as an Elitist Equilibrium (EE ).

De..nition 1 Wede. neSingle Class Equilibrium (SCE) as onein which high-skill types are willing to work with low-skill agents. We de. ne Elitist Equilibrium (EE) as one in which the high-skill agents refuse the low-skill agents.

De..nition 2 Given $N(t)$, the number of agents (workers or employers), and $1 / 4 t$ ), the proportion of type $\mathrm{H}$ on the market at period $\mathrm{t}$, we de .ne Steady StateEquilibrium by two conditions: (i) $N(t+1)=N(t)=N$ and (ii) $1 / 4 t+1)=1 / 4 t)=1 / 4$ We further impose consistency between agents strategies and the equilibrium.

Single Class Equilibrium We ..rst analyze the conditions under which high-skilled $(H)$ agents decide to work with the low-skilled $(L)$.

The total discounted utility of accepting $L$ is:

$$
U={ }_{t=0}^{*^{1}} \pm\left(1_{i}-\right)^{t} x_{l}=\frac{x_{l}}{11_{i} \pm\left(1 i^{-}\right)}
$$

where \pm denotes the discount factor, and $\left(1_{\mathrm{i}^{-}}{ }^{-}\right)$the probability to be alive in the next period.

Let $\mathrm{U}_{\mathrm{h}}$ denotethe expected discounted utility of an $\mathrm{H}$ unemployed worker (employer). An agent $\mathrm{i}$ has a probability ${ }^{\circledR}$ to meet somebody in the search market. If this is the case, he will obtain an utility $x_{h}$, with probability $1 / 4$ and $x_{1}$, with probability $(1 ; 1 / 4$. With the probability $\left(1_{i} \AA\right.$ ), this will not be the case, and the agent will try again to match next period.

Hence, $U_{h}$ satis. es

$$
U_{h}=\frac{\circledast\left[1 / x_{h}+\left(1 i^{1 / 4} x_{l}\right]\right.}{1 i \pm\left(1 i^{-}\right)}+ \pm\left(1 i^{-}\right)(1 ; @) U_{h}
$$


This equation can be rewritten as follows:

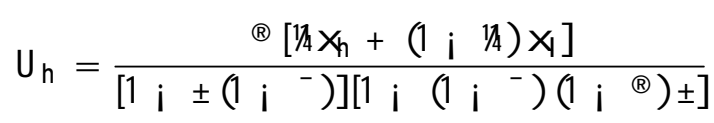

To guarantee existence, we need the intertemporal utility of an $\mathrm{H}$ agent accepting $\mathrm{L}$ to be bigger than the utility of continuing to search. This requires that:

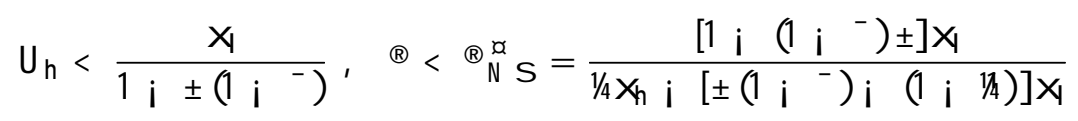

In the SCE, average unemployment duration is the same for both types of agents and is equal to $1=\AA$.

$N(t)^{1 / 4 t} t$ describes the number of $H$ agents at time $t$. In equilibrium, $N(t)$ and $N(t)^{1 / 4 t}$ ) are expected to evolve according to:

$$
\begin{aligned}
& \mathrm{N}(\mathrm{t}+1)=\left(1 \mathrm{i} \mathbb{B}^{-}{ }^{-}\right) \mathrm{N}(\mathrm{t})+. \\
& \left.\left.\mathrm{N}(\mathrm{t}+1)^{1 / 4} \mathrm{t}+1\right)=\left(1 ;{ }^{\circledR} \mathrm{i}^{-}\right) \mathrm{N}(\mathrm{t})^{1 / 4} \mathrm{t}\right)+{ }^{\prime},
\end{aligned}
$$

Hence, a stationary state occurs if and only if

$$
\mathrm{N}=\frac{\partial}{(\mathrm{B}+} \text { and } 1 / 4=
$$

Elitist Equilibrium We now look at dixerent strategies by the agents. Suppose that $\mathrm{H}$ types will not sign a contract with the $\mathrm{L}$ types. If this is an equilibrium, $\mathrm{L}$ types must be willing to marry each other, that is, when the $\mathrm{H}$ worker refuses to work with the $L$ type, the $L$ type is obliged to work with the $L$ agents. The average unemployment duration for the $\mathrm{H}$ is therefore $1=\mathbb{R} / 4$ whereas the average unemployment duration for the $L$ is $1=B(1 ; \quad 1 / 4$.

Equilibrium exists if and only if $H$ types prefer to reject $L$ types, so that

$$
U_{h}>\frac{x_{l}}{1 i \pm\left(1 i^{-}\right)}
$$

where

$$
U_{h}=\frac{\circledast\left[1 / x_{h}\right]}{1_{i} \pm\left(1 i^{-}\right)}+ \pm\left(1 i^{-}\right)\left[\otimes\left(1 i^{1 / 4}+(1 ; \quad \Theta)\right] U_{h} ;\right.
$$

which requires that:

$$
\circledR>\mathbb{B}_{S}^{M}=\frac{\left[1_{i}\left(1_{i}{ }^{-}\right) \pm x_{l}\right.}{\left.1 / 4^{\prime}\right)\left[\left(x_{h} i x_{l}\right) i_{i}\left[1_{i} \pm\left(1_{i}{ }^{-}\right)\right] x_{l}\right]}
$$


Given these equilibria, $N(t)$ and $N(t)^{1 / 4 t}$ ) are now expected to evolve according to:

$$
\begin{aligned}
& \left.\left.\left.N(t+1)=N(t)[@ / 4 t)(1 ; \quad 1 / 4 t))+{ }^{\circledR}(1 ; \quad 1 / 4 t)\right) 1 / 4 t\right)+1 ; \quad{ }^{i}{ }^{-}\right]+, \\
& \left.\left.\left.N(t+1)^{1 / 4 t} t+1\right)=N(t)^{1 / 4 t}\right)\left[\left({ }^{1} i^{1 / 4 t} t\right)\right)+1 ;{ }^{\circledR} i^{-}\right]+,
\end{aligned}
$$

In this case the stationary state is as follows:

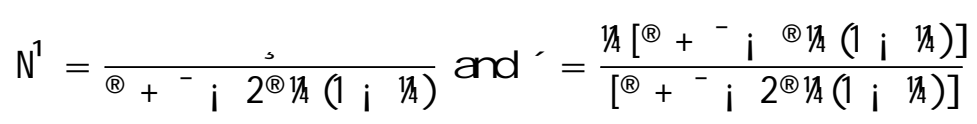

where ' is the exogenous proportion of types $\mathrm{H}$ fowing into the market at any period and $1 / 4$ is his endogenous value.

Straightforward algebra shows that a solution $1 / 42[0 ; 1]$ al ways exists and is unique. Furthermore, if ' $<0: 5$ then $1 / 42\left[{ }^{\prime} ; 0: 5\right]$; while if ${ }^{\prime}>0: 5$ then $1 / 42\left[0: 5 ;^{\prime}\right]$.

There are parameter values where both steady-state equilibria exist.

Theorem 1 (Burdett-Coles): both a SCE and an EE exist if and only if $0<{ }^{\prime}<0: 5$, and

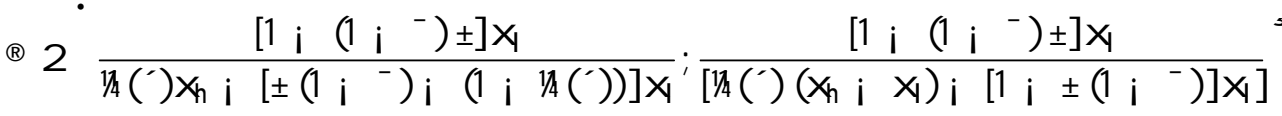

where $\left.1 / 4^{\prime}\right)>{ }^{\prime}$.

Proof. Se Burdett and Coles (1997)

If the proportion of $\mathrm{H}$ (high type) agents goes beyond 0:5, the labor market becomes segmented since the optimal strategy for $\mathrm{H}$ is to refuse to sign a contract with $\mathrm{L}$ agent. Then, the probability of ..nding a partner decreases for both types of agents. Neverthe less, the rise in unemployment duration axects $\mathrm{L}$ more than $\mathrm{H}$. In fact, the situation of $\mathrm{L}$ types worsens both because of the smaller probability to match somebody and the lower level of human capital of their potential partner.

\section{Search Equilibrium with Temporary M atching}

\subsection{Temporary $M$ atching without Delay Cost}

We .r.st characterize the equilibria when the agent, after the temporary employment, comes back to the market immediately without waiting (that is, in the absence of a delay cost). 
With the additional strategy "going into the temporary employment", the set of possible equilibria is enlarged, moreover, whilein the previous section the $L$ agents accept passively the choice of the $\mathrm{H}$ agents, here, on the contrary, they can react strategically.

As in the previous section there are two types of equilibria, characterized by whether $\mathrm{H}$ types are willing to work with $\mathrm{L}$ types (SCE) or are not willing to do so (EE).

We now also have to check if it is the case that the $L$ agent al ways prefers to reject another $\mathrm{L}$ type in the search market, with the hope of meeting an $\mathrm{H}$ type in thetemporary market.

De..nition 3 We de.ne Temporary Unskilled Equilibria(TUE) as the equilibrium in which low-skill types match exclusively in the temporary market.

Within each class of possible equilibria we have to consider the possibility for the agents to go to the temporary agency (TA) if they do not match in the search market.

We thus have the following possible equilibria:

SCE

1) $\mathrm{L}$ and $\mathrm{H}$ go to the temporary agency (TA);

2) $L$ goes to the TA (not $H$ );

3) $\mathrm{H}$ goes to the TA (not $L$ );

4) nobody goes to the $T A$;

EE

1) $\mathrm{L}$ and $\mathrm{H}$ go to the temporary agency;

2) $L$ goes to the TA (not $H$ );

3) $\mathrm{H}$ goes to the TA (not $L$ );

4) nobody goes to the TA;

TUE

1) $\mathrm{H}$ goes to the $T A$;

2) $\mathrm{H}$ does not go to the TA.

Theorem 2 In a labor market with a simultaneous presence of a search market and a temporary, only two types of equilibrium exist. EE-2 exists i $\$, \$ \frac{a}{1}$, and TUE-1 exists i $\$, \phi_{2}^{\alpha}$ and $\phi \cdot \phi_{3}^{\alpha}$ where:

$2 \Phi_{1}^{\alpha}=\frac{\left(1_{i} C^{2}+\otimes C^{2} / / 4\right)}{\left.\left[l_{i} C^{2}+B C C^{1 / 2} / 2+C\right)\right]}$

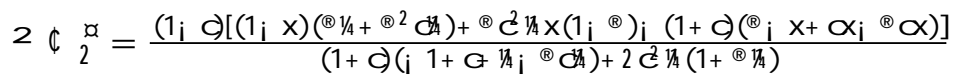

$2 \phi_{3}^{\alpha}=\frac{\left.x\left(1_{i} c^{2}\right)+\otimes c^{1} / 4 x+c x_{i} 1\right)}{\otimes c^{1} / 4}$. With $c= \pm\left(1_{i}{ }^{-}\right)$and $x$ is the expected value in the temporary employment. 
Proof. Se the Appendix 2

For these equilibria, we can calculate the Steady State value of the unemployed (vacancies) and of the unemployed (vacancies) that have type $x_{h}$.

The following conditions characterize the Steady State equilibria TUE-1 and EE-2, where $\hat{N}$ is the Steady State value of the unemployed (vacancies) and $\mathbf{1} / \mathbf{4}$ the Steady State proportion of the $\mathrm{H}$ types of this value.

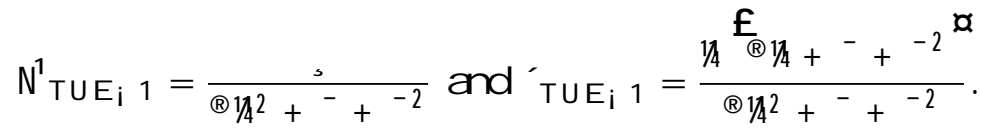

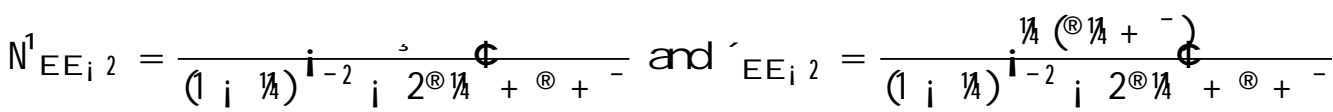

Straightforward algebra shows that a solution $1 / 4 U_{E_{i} 1}$ and $1 / 4 E_{i} 22[0 ; 1]$ always exists and is unique, furthermore, 8', 1/4 $\mathrm{UE}_{\mathrm{i}} 12\left[0 ;{ }^{\prime}\right]$ and $1 / \mathbb{4} \mathrm{E}_{\mathrm{i}} 22\left[{ }^{\prime} ; 1\right]$.

P roposition 3 If $^{\prime}>0: 5$, the situation of both types of agents is improved in the model with TA without delay cost (model II), when compared to the model without TA (model I)

In model II, the $\mathrm{H}$ type always refuses the $\mathrm{L}$ type, for every ': On the contrary, in mode $\mathrm{I}$, the $H$ agent refuses a $L$ type only if ' $>0: 5$. Hence $L$ types are worse ox for $'<0: 5$ and indixerent for' $>0: 5$.

In any case, the number of worker (employers) looking for a job (worker) will belower: both steady state equilibria (equations (6) and (7)) of model II will lead to a smaller $N$ (less unemployednvacancies), than in the EE (equation (4)) of model $\mathrm{I}^{2}$. Therefore, the situation of both types of agents is always improved.

\subsection{Temporary M atching with Delay Cost}

Theorem 5 shows us that the introduction of the possibility to form temporary labor relations could have positive exects in the labor market because unemployment is lower. Nevertheless, this improved situation is exploited unilaterally from the more quali..ed agents, who change their strategies in that they refuse systematically to sign a contract with the less endowed agents. This comes about for the hypothesis that once the temporary matching is dissolved, the agents can form another (temporary or life) match without delay. Why should a skilled agent accept an unskilled one for life if he can wait

\footnotetext{
${ }^{2}$ Notice that if ' $>0: 5$ (model I) only EE holds. See Theorem 3 for more details.
} 
for meeting a preferred agent and, in the meantime, try the temporary market where he has a strictly positive probability of being matched to a skilled individual?

In this section, we maintain the hypothesis that if the agent does not match in the search market, he can go to the temporary market. But now if he matches in the TA, he has to pay a cost of delay: after the temporary matching the agent has to wait for one period out of the market before searching again.

Theorem 4 in a labor market with a simultaneous presence of a search and a temporary market with delay cost, SCE-1 exist if and only if $\phi \cdot \phi_{1}^{\text {ax }}$ and $\phi \cdot \phi_{2}^{\text {ax }}, \mathrm{EE}-1$ ix

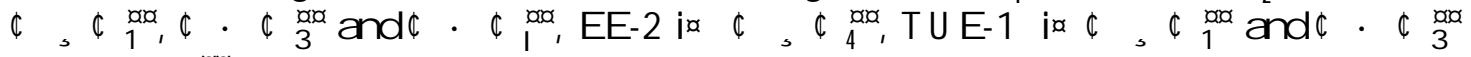
and $\phi, \phi_{1}^{\text {pad }}$. With

$2 \phi_{1}^{m a x}=\frac{\left(1_{i} c^{3}\right) i x\left(1 i_{i} c^{2}\right)}{\left(1_{i} c^{3}\right)+2 \otimes c^{3} 1_{4}}$

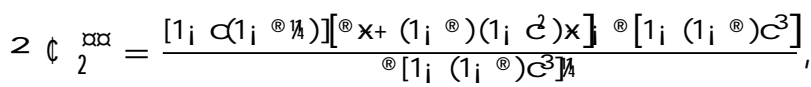

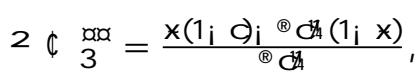

$2 \Phi_{4}^{\cos }=\frac{\left(1_{i} c\right)}{\left(1_{i} \mathrm{C}+2 \mathbb{B} C^{1 / 4} / 4\right.}$

${ }^{2} \phi_{1}^{\text {pod }}=\frac{1+c+c^{2} i x(1+c)}{1+c+c^{2}}$. Where $c= \pm\left(1_{i}{ }^{-}\right)$and $x$ is the expected value in the temporary employment.

Proof. Se Appendix 3

The following conditions characterize the Steady State equilibria SCE-1, EE-1, EE-2 and TUE-1:

$$
\begin{aligned}
& N_{\mathrm{SCE}} \mathrm{i}_{1}=, \neq\left({ }^{-}+^{-}++^{-2}+^{-3}\right) \text { and }{ }^{\prime}=1 / 4
\end{aligned}
$$

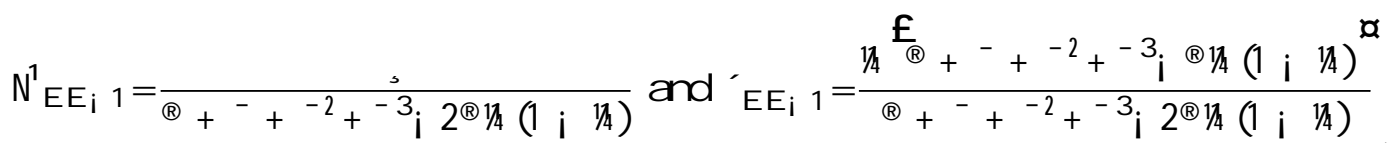

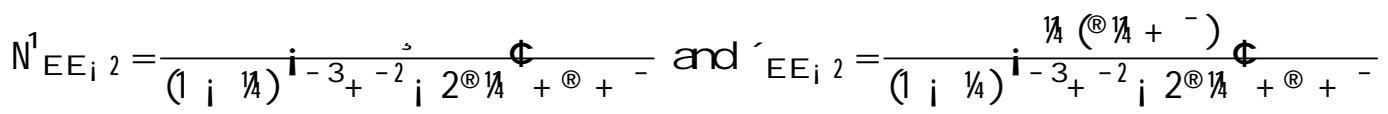

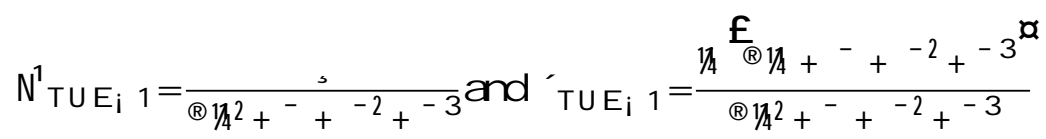

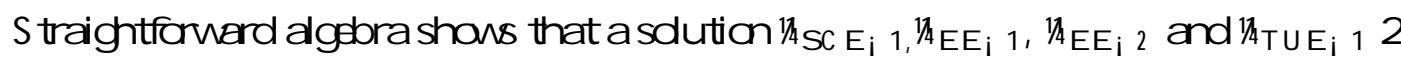
[0; 1] always exists and is unique for each equilibria. Furthermore: 
$28^{\prime}, 1 / \mathbb{E} E_{i} 22\left[{ }^{\prime} ; 1\right]$ and $1 / 4 U_{E_{i} 1} 2\left[0 ;{ }^{\prime}\right] ;$

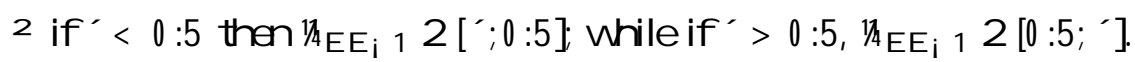

If we consider a cost in the TA (mode III), the situation becomes more complex in that we now have more equilibria that are possible Now, if an agent decides to enter the temporary market, he has to pay a cost (delay cost).

The positive exect of the TA in $\mathrm{N}$ remains unchanged: indeed, in mode III, EE-2 and TUE-1 will lead to a smaller $N$ (less unemployednvacancies), than the respective ones emerging from the model II (cfr. (10) with (7) and (11) with (6)).

Moreover, the $\mathrm{H}$ agent cannot fully exploit the improved situation in the market, because of the cost for entering the temporary market: an $H$ agent accepts a $L$ one if $1 / 4$ and $\$$ are suф ciently low.

\section{Comparative Statics}

In the former sections we have characterized the equilibria of three models. In each model, the market is composed of either one or two groups, depending on the ec ciency of the matching process ( $(\mathbb{B})$, the proportion of quali..ed agents ( $1 / 4$ and the degree of heterogeneity of the population $(\$)$. This section will analyze the impact of the variations of these terms on unemployment and on the segmentation degree.

\subsection{Search E $₫$ ciency}

A common view about unemployment is that an increase in the eq ciency of the matching process has al ways a positive impact on unemployment $\hat{N}$, where $\hat{N}$ denotes the Steady State number of workers (employers) searching for an employment (worker). Model I, without TA, suggests that a rise in eq ciency matching, ${ }^{\circledR}$, may have adverse exects on unemployment, because it modi. .es matching strategies. Therefore, a rise in $₫$ can have either a positive or a negative exect on the rate of unemployment.

In the SCE case, $N$ (see equation (2)) decreases with ${ }^{\circledR}$.

$$
\frac{@ \sqrt{ }}{@ \circledast)}=\frac{i}{\left(囚+^{-}\right)}<0
$$

In the EE case, $N$ (see equation (4)) also decreases with ${ }^{\circledR}$

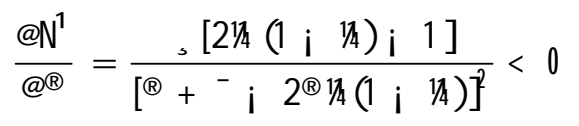

Theinteresting point is what happens if we pass from an equilibrium to the other. We know that the labor market will be divided (segregated) in two segments when ${ }^{\circledR}>{ }^{8}{ }_{S}$ 
(see condition (3)) and will stay uni..ed (non segregated) if ${ }^{\circledR}<\mathbb{R}_{\mathrm{N}} \mathrm{S}$ (see condition (1)). An increase in ${ }^{\circledR}$ over $\mathbb{B}_{S}^{M}$ and $\mathbb{R}_{\mathrm{N}}^{\mathrm{N}}$ increases the willingness of the $\mathrm{H}$ agents to match between them segregating the $L$ types. Clearly, $\hat{N}$ in EE is bigger than $\hat{N}$ in SCE (see conditions (4) and (2) $)^{3}$. Hence, we may have an increase in ${ }^{\circledR}$ followed by an increase in Steady State unemployment.

If weintroduce a centralized TA, without delay cost (mode II), the situation changes. An increase in ${ }^{\circledR}$ has a positive exect on unemployment both in EE-2 (see equation (7)) and in TUE-1 (see equation (6)):

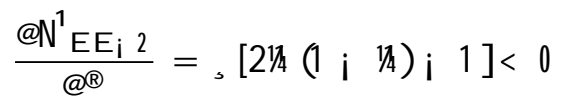

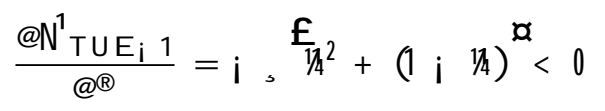

Moreover, this increase in the et ciency of the matching process tends to decrease the critical level of heterogeneity $\Phi_{1}^{\alpha}$ beyond which a EE-2 exists

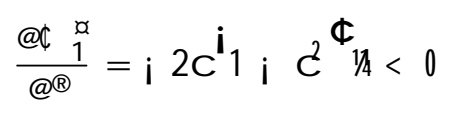

It is easy to show that, if ${ }^{-}$is small enough, $\hat{N}_{T U E_{i} 1}(6)$ is always greater than $\mathrm{N}_{\mathrm{EE}_{\mathrm{i}} 2}$ (7). Unemployment will be lower in the EE-2 (7) with respect to TUE-1 (6).

In the search market, the matching strategies of the skilled agents will not change depending on ${ }^{\circledR}$. this market will be always segmented. Instead, the strategies will depend on ${ }^{\text {in }}$ the temporary market: the skilled agents have to decide whether to enter or not into this market (the less skilled agents al ways enter). If they do so, unemployment will be higher because of the greater instability of the market. The reason is that ..xedduration contracts are temporary by nature, so their lifetime is much shorter than that of the regular, long-term ones.

The dixerences between the two equilibria is not so important. We can just pass from an economy (EE-2) where the situation of the less skilled agents is worse because they can not meet higher skilled agents (indeed the more skilled agents do not enter the temporary market) to an economy (TUE-1) where the situation of the less skilled agents is improved (both the more and the less skilled agents enter the temporary market) but the unemployment is lower.

With a delay cost in the TA (mode III), thesituation becomes more complex because four equilibria are possible Wecan easily show that an increase in ${ }^{\circledR}$ has al ways a positive exect on each steady state unemployment ${ }^{4}$ as long as it does not force the change from

${ }^{3}$ See also Rioux (1995) for general results in the basic model without temporary market.

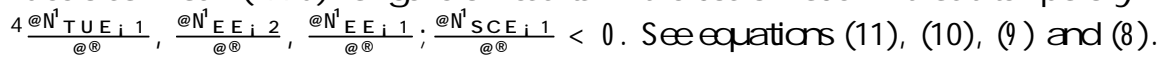


one type of equilibrium to another. If this happens we could pass from an equilibrium where the steady state unemployment is low (like the SCE-1) to an equilibrium with high unemployment (like TUE-1). We can easily verify easily that, if ${ }^{-}$is small enough, $N_{T_{U E} i_{1}}^{3}>N_{E_{E_{i}} 2}>N_{E_{E_{i} 1}}^{3}>N_{\text {SCE }}$ 1.

A better ec ciency in the matching leads the more skilled to reject the less skilled agents in the search market ${ }^{5}$, and to not enter the temporary market ${ }^{6}$.

The less skilled agents respond by making matches among themselves ${ }^{7}$ but they continue to stay in the temporary market with the hope of meeting a more skilled agent 8 .

\subsection{Proportion of Educated}

For some economists, the answer to the depression in the labor market is to increase the proportion of educated people.

As we pointed out in Theorem 1, if ', the proportion of high-skill $(H)$ agents who enter the market at each period goes beyond 0:5, the market becomes segmented sincethe optimal strategy for the $\mathrm{H}$ agents is to segregatefrom therest of theeconomy. An increase of ', if this critic value is attained, has a negative exect on unemployment. On the contrary, if we do not go beyond this value, this increase has no exect on unemployment (see equation (2)).

If ' is al ready bigger than 0:5, an increase in ' (and so in $1 / 4$ has a positive eaect (see equation (4)):

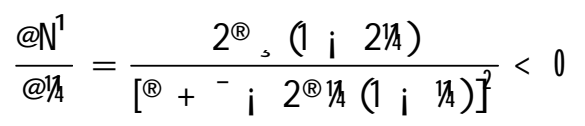

If we turn to the model with TA, the change in this proportion can have some exects on unemployment.

An increase in the proportion of the educated, $1 / 4$ has the same exect as the increasing in the search ec ciency, $\AA_{\text {, }}$ as far as switching between types of equilibrium it concerned:

2 in the model with TA but without delay cost (mode II), it induces a decrease

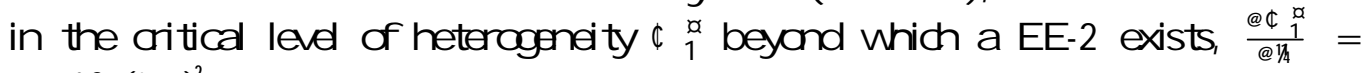
$\frac{i 2 \otimes C\left(1_{i} C\right)^{2}}{\left[1_{i} C^{2}+B C(1 / 42+C)\right]^{2}}<0$;

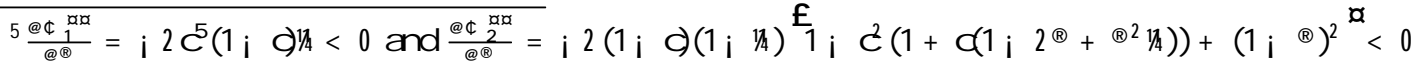
(in the SCE-1).

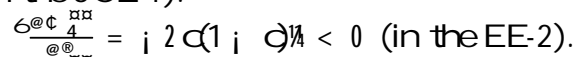

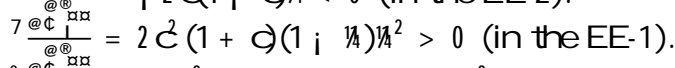

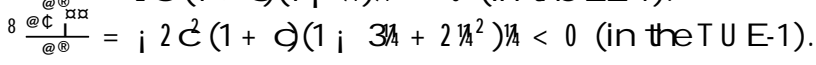


2 in the model with a delay cost (model III) it implies the same qualitative results on the various critical levels of heterogeneity.

However, unlike change in ${ }^{\circledR}$, rises $1 / 4$ may have ambiguous exects on the rate of unemployment in each type of equilibrium. In the SCE-1 this variation has no exect (equation (8)). In the EE-1 case (equation (9), and in the TUE-1 case (both in model II and in mode III, see equations(6) and (11)) the exect is al ways positive. Finally in EE-2

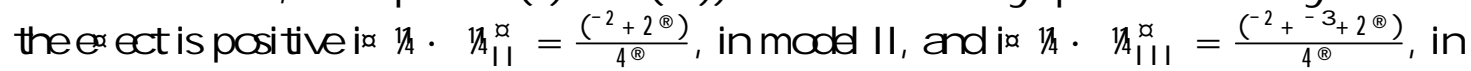
mode III (equations (7) and (10)).

\subsection{Degree of Heterogeneity}

We are now interested in the impact of a change in $\phi$, where the parameter which measures the dixerence in human capital endowment between high-skilled and low-skilled agents. In each mode, this has no implications for the Steady State unemployment under each type of equilibrium. There can be some exect, in the line with what happens if we vary $\AA$ and $1 / 4$ only if we pass from an equilibrium to another one.

In model I, the impact of an increase in inequality clearly appears from equations (1) and (3)

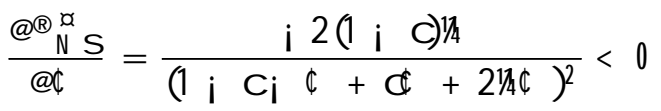

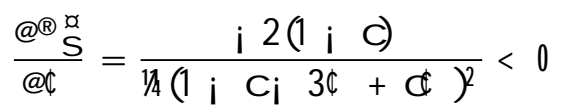

That is, for given $c$ and $1 / 4$ an increase in the leve of heterogeneity tends to reduce the critical level $\mathbb{R}_{\mathrm{N}}^{a}$, under which the labor market does not segregate, and $\mathbb{B}_{5}^{\prime}$, beyond which the labor market segregates. Since $L$ agents become less desiderable than potential partners, a more unequal distribution of human capital in the population raises the willingness of the good agents to segregate.

In mode II, the agents strategies will change with the critical values of the degree of heterogeneity $\phi_{1}^{\alpha}, \Phi_{2}^{\alpha}$ and $\phi_{3}^{\alpha}$. We have three possible situations:

2 if $\phi>\Phi_{1}^{\alpha}$ and $\phi<\Phi_{2}^{\alpha}$ (or $\phi>\phi_{3}^{\alpha}$ ), only the EE-2 exists;

2 if $\phi<\phi_{1}^{\alpha}, \Phi>\phi \frac{\alpha}{2}$, and $\phi<\phi_{3}^{\alpha}$; only the TUE-1 exists;

2 if $\phi>\phi \frac{\alpha}{1}, \Phi>\phi \frac{\alpha}{2}$, and $\phi<\phi \frac{\alpha}{3}$, both the EE-2 and TUE-1 exist.

\footnotetext{
${ }^{9}$ These values are been de. ned in the section 4.1.
} 
An increase in $\phi$ over $\phi_{1 ; 2 ; 3}^{d}$ augments the willingness of the more skilled agents to match between them, which causes the segregation of the less skilled agents not only in the search market but also in the temporary one, since skilled individuals will then refuse to enter the latter. Consequently, the willingness of the less skilled to match between them will be higher.

In mode III, the impact of an increase in inequality appears from Theorem 4. It is not easy to analyze it because there are too much critical values. Moreover it seems clear that an increase in $\phi$ over $\phi_{1}^{x 10}$ augments the willingness of the more skilled to be more selective. Consequently the conditions to the occurence of SCE-1 are less easily satis. ed, and EE-2 and TUE-1 are more easily satis. ed.

In addition, an increase in $\phi$ over $\phi_{1}^{\alpha}$ augments the willingness of the low-skilled to match, even if temporarily, with the more quali..ed agents: the TUE-1 occures under bigger and the EE-1 under smaller ranges of parameter values .

\section{Conclusions}

The depression in the labor market in the developed countries is explained by the fact that there are too many unskilled workers and too less skilled workers for the existing technol ogy at thetime To solvethis disequilibrium the level of education should beaugmented. If we increase suф ciently the education of the population, the unskilled workers could be employed because they can take advantage of the decreasing competition in the market where they search. Nevertheless, as the ..rst model suggests, the higher proportion of skilled workers can lead them to reject the unskilled workers because they prefer to form more homogenous productive unities.

The introduction of a temporary market has the exect of reducing the key importance of the search eq ciency and the proportion of educated for the determination of the equilibrium. Moreover, if on the one hand it could have a positive impact on unemployment, on the other hand it implies less opportunities for less skilled agents. The latter can just try to stay as long as possible in the temporary market in the hope of forming a temporary match with a high-skilled agent.

This policy could loose its positive impact on unemployment if additional appropriate policies were not carried out. The possibility of free entry in this market could produce great instability in the labor market, because of the temporary nature of these kinds of contracts. If we introduce an entry cost, we can keep the positive exects of this policy and people will enter this market only if they really need.

Moreover, the best way to improve low-skilled agents' welfare, as Saint-Paul (92) and Rioux (95) suggest, is not necessarily to give a fraction of them education and change them into high-skilled: it can even lead to a perverse exect if this increase in the number

\footnotetext{
${ }^{10}$ The critical values have been de. ned in the section 4.2.
} 
of $\mathrm{H}$ agents is suф cient to encourage them to segregate $\mathrm{A}$ better way to improve the situation of the poor would be to give all of them a higher quality education: as human capital heterogeneity decreases, $\mathrm{H}$ agents will be more willing to accept less educated agents as partners.

This policy should always be accompanied by a temporary market regulation to avoid that the improvement of quality education loses its positive exect. 


\section{Appendix 1. Proof of Theorem 2}

We establish Theorem 5 by a series of lemmas.

Lemma 1 The only candidate equilibria are SCE-1, EE-1, EE-2 and TUE-1.

We can exclude all other equilibria con. .gurations. SCE-2 and SCE-3 are excluded because with the assumption that a high-type worker will accept the low-type in the search market (SCE) and that the utility to be unmatched is zero, a $\mathrm{H}$ type will al ways prefer to go to the temporary market if he does not match in the search market: SCE-2 does not exist. We can use the same argument for the $L$ type in SCE-3.

EE-3 is excluded because with the assumption that the temporary agency matches the agents at random, if the $\mathrm{H}$ types are in this market, a $\mathrm{L}$ agent will enter thetemporary market too: the EE-3 never exist.

TUE-2 is excluded because if the $H$ type does not go to the TA, the $L$ agents will accept each other in contact in the search market: the TUE-2 never exist.

Finally, we can restrict the possible equilibria by eliminating the SCE-4 and EE-4 because they are dominated respectively by SCE-1, for both types of agents, and by EE-2 for $L$ types agents.

SCE-1 is preferred to SCE-4 if and only if

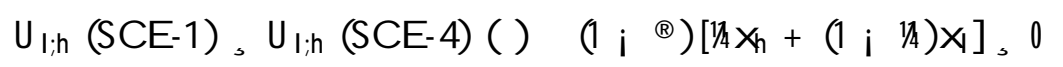

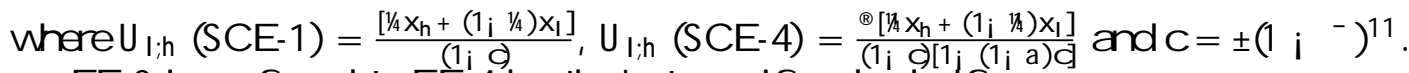

$E E-2$ is preferred to $E E-4$ by the $L$ types if and only if

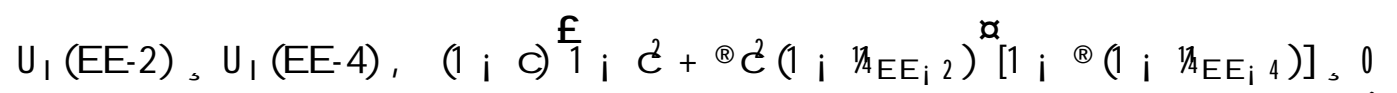

where $1 / \mathbb{E} E_{i} 2$ and $1 / \mathbb{E} E_{i} 4$ are the Steady State proportion of $H$ agents respectively in

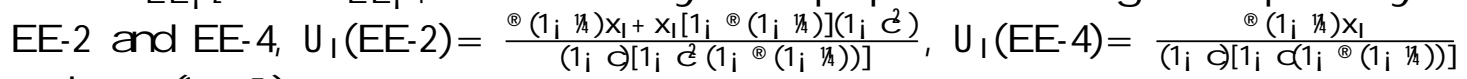
and $\left.\mathrm{C}= \pm 1 \mathrm{i}^{-}\right)$.

The conditions (12) and (13) are always satis. ed because the term on the left hand side is always positive

We now compute the equilibrium utility. Let us de. ne $U_{h 1}, U_{h 2}, U_{h 3}, U_{h 4}$ for the $H$ type, and $U_{l}, U_{\text {ta }}$ for the $L$ type:

\footnotetext{
${ }^{11}$ The Steady State proportion of $\mathrm{H}$ agents, $1 / 4$ are exactly the same in the SCE-land SCE-4.
} 


\begin{tabular}{|c|c|}
\hline$U_{h}\left(x_{1}, T A\right)$ & 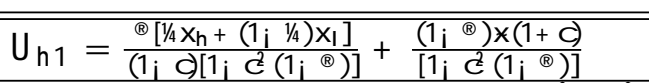 \\
\hline$U_{h}\left(\right.$ no $\left.x_{1}, T A\right)$ & 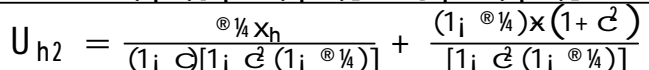 \\
\hline$U_{h}\left(x_{1}\right.$, no TA $)$ & 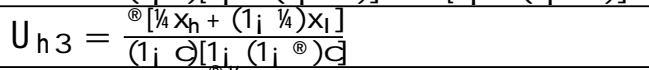 \\
\hline$U_{h}\left(\right.$ no $x_{1}$, no TA $)$ & 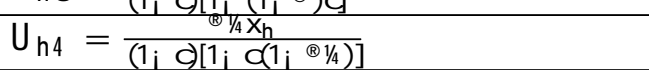 \\
\hline $\mathrm{U}_{1}\left(\mathrm{x}_{1}, \mathrm{TA}\right)$ & 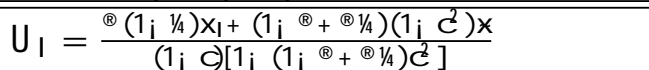 \\
\hline$U_{1}\left(\right.$ no $\left.x_{1}, T A\right)$ & $\mathrm{U}_{\mathrm{ta}}=\frac{x(1+\mathrm{c})}{\left(1_{\mathrm{i}} \mathrm{c}^{2}\right)}$ \\
\hline
\end{tabular}

where $x$ is the expected value in the TA and $c= \pm\left(1_{i}{ }^{-}\right)$.

Lemma 2 SCE-1 ( $L$ and $\mathrm{H}$ go to the TA) never exists.

The utility of a $\mathrm{H}$ type in the SCE- 1 is $U_{h 1}$, where $x=\left[\begin{array}{ll}1 / x_{h} & +\left(1_{i}{ }^{1 / 4} x_{l}\right.\end{array}\right]$.

In computing the utility of the agents, we consider the possibility of going to the temporary agency. After the matching in the search market the proportion of agents unmatched in SCE will be unchanged.

This is an equilibrium if and only if no possible deviation from the equilibrium is pro.table. This condition requires that $U_{h 1}$ is bigger than the following utilities of deviating: $\mathrm{U}_{\mathrm{h} 2} ; \mathrm{U}_{\mathrm{h} 3} ; \mathrm{U}_{\mathrm{h} 4}$.

If we look just at the .r.st of these three conditions, we ..nd:

$$
U_{h 1}>U_{h 2}() \quad \mathbb{R} / 4\left(1 ; 1 / 4\left(x_{1} ; x_{h}\right)\right]>0
$$

This condition is never true

Lemma $3 \mathrm{EE}-1$ ( $\mathrm{L}$ and $\mathrm{H}$ go to the TA) never exists.

Equilibrium requires $\mathrm{H}$ to refuse to sign a contract with $\mathrm{L}$ and to go to the TA, and $\mathrm{L}$ type to accept another $\mathrm{L}$ type in the search market.

This is never an equilibrium because the unemployed $L$ prefers to deviate from the equilibrium by refusing the $L$ vacancies in order to go directly to theTA. The equilibrium exists if and only if

$$
U_{1}>U_{\text {ta }}() \otimes\left[\left(1 ; 1 / 4\left(x_{1} ; \quad x\right) ; 2 c^{1 / x_{1}}\right], 0\right.
$$

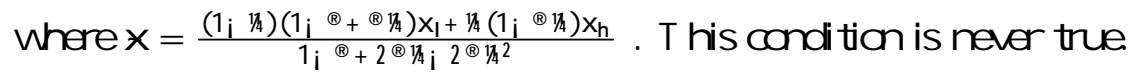

Lemma 4 EE-2 ( $L$ go to TA, H never go to the TA) exists ix $\pitchfork>\Phi_{1}^{\alpha}$, where $\Phi_{1}^{\alpha}=$ $\frac{\left(1_{i} c^{2}+B C^{21} / 4\right)}{\left[1_{i} C^{2}+B C^{1 / 42+C)}\right]}$. 
Equilibrium conditions are $\mathrm{U}_{\mathrm{h} 4}>\mathrm{U}_{\mathrm{h} 3} ; \mathrm{U}_{\mathrm{h} 1} ; \mathrm{U}_{\mathrm{h} 2}$.

We can demonstrate easily that $U_{h 2}$ is al ways bigger than $U_{h 1}$. In fact $U_{h 2}, U_{h 1}()$ $2{ }^{2} c^{2} \phi(1 ; 1 / 41 / 4,0$. So we can diminate the second condition. The new condition will be: $U_{h 4}>U_{h 3} ; U_{h 2}$.

2 The .r.st condition is

$$
U_{h 4}>U_{h 3}() \quad \phi, \phi^{\infty}=\frac{(1 ; c)}{\left(1 ; c+2 \mathbb{B} C^{1} / 4\right.}
$$

2 the second condition is

$$
U_{h 4}>U_{h 2}() \quad \phi, \quad \phi_{1}^{\infty}=\frac{i_{1 i} c^{2}+B c^{2} 1 / 4}{\left.\left[1 ; c^{2}+B C^{1 / / 4}+c\right)\right]}
$$

where $x=x_{1}$.

We can show that $\phi^{x} \cdot \phi_{1}^{\alpha}$. So, we can eliminate $\phi^{x}$. We thus have to consider just the second condition.

Lemma 5 TUE-1 exists i $\phi>\phi_{2}^{\alpha}$ and $\phi>\phi_{3}^{\alpha}$ where

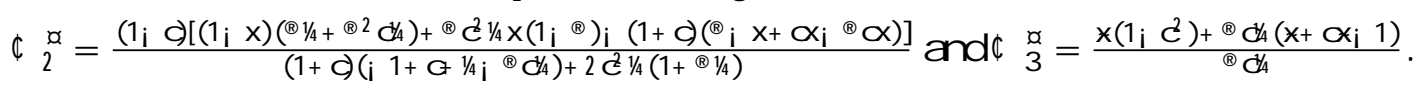

We have an equilibrium if and only if $\mathrm{H}$ refuses to sign a contract with $\mathrm{L}$ and wishes to go to the TA, and if and only if an $L$ type does not accept another $L$ type in the search market.

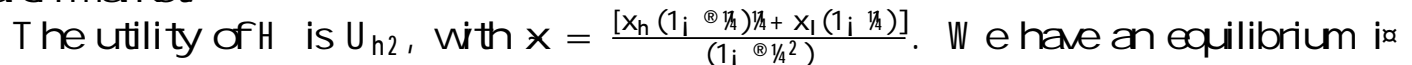
$U_{h 2}>U_{h 1}, U_{h 3}, U_{h 4}$.

2 The .r.st condition is

$$
U_{h 2}>U_{h 1}() \quad\left(1 ; \quad 1 / A\left(1 ; c^{2}\right)\left(* ; \quad x_{1}\right)+2 \mathbb{B} C^{2} \Phi^{1 / 4}, 0\right.
$$

2 The second condition is

$$
U_{\mathrm{h} 2}>U_{\mathrm{h} 3}() \notin, \Phi_{2}^{\mathrm{x}}
$$

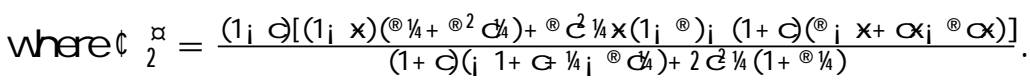

2 The third condition is

$$
U_{h 2}>U_{h 4}() \notin, \$_{3}^{x}
$$

where $\phi_{3}^{\alpha}=\frac{x\left(1_{i} c^{2}\right)+B c 1 /(x+c x i 1)}{B c x}$ 
For the $L$ type we have an equilibrium ix:

$$
U_{t a}, U_{1}() \otimes\left[\left(1 ; \quad \mathbb{A} / 4\left(x ; x_{1}\right)+2 c^{1 / x} x_{1}\right], 0\right.
$$

The conditions (15) and (16) are always true for any parameters value. 


\section{Appendix 2 proof of theorem 4}

We establish Theorem 7 by a series of lemmas.

Lemma 1 The only candidate equilibria are SCE-1, EE-1, EE-2 and TUE-1.

We can exclude all other equilibria con..gurations. SCE-2, SCE-3, EE-3 and TUE-2 are excluded for the same arguments as in the previous model (see Appendix 2, Lemma $1)$.

Moreover, SCE-4 and EE-4 are excluded because they are always dominated respectively by SCE-1, for both types of agents, and by EE-2 for L types agents.

SCE- 1 is preferred to SCE-4 if and only if

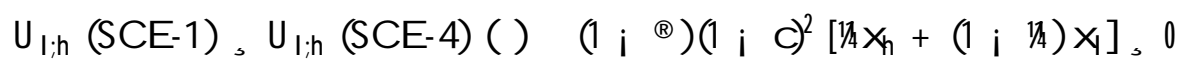

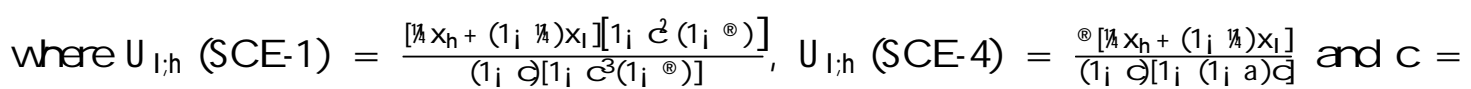
$\#\left(1_{i}{ }^{-}\right)^{12}$.

$E E-2$ is preferred to EE-4 if and only if

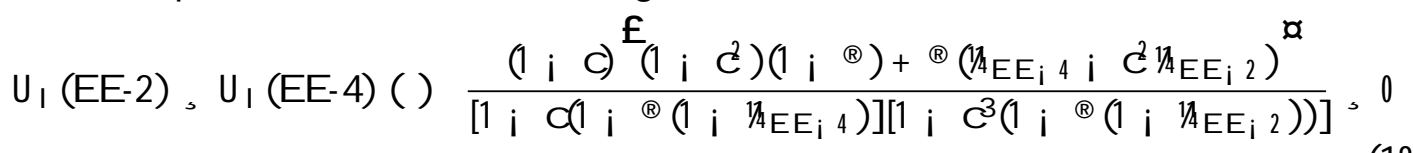

where $1 / 4 E_{i} 2$ and $1 / \mathbb{4} E_{i} 4$ (the Steady State proportion of $H$ agents respectively in $E E-2$

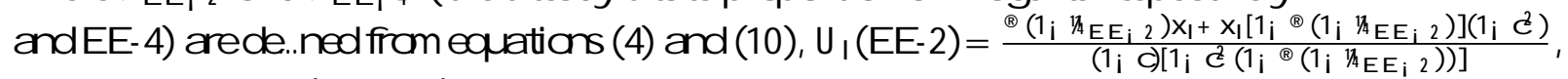

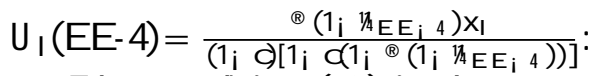

The condition (18) is always true ix:

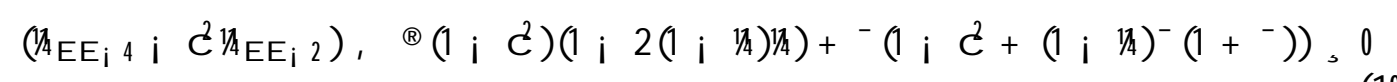

the conditions (17) and (19) are al ways true.

We now compute the equilibrium utility. Let us de. ne $U_{h 1}, U_{h 2}, U_{h 3}, U_{h 4}$ for the $H$ type, and $U_{1}, U_{\text {ta }}$ for the $L$ type:

\footnotetext{
${ }^{12}$ The Steady State proportion of $\mathrm{H}$ agents, $1 / 4$ are exactly the same in the SCE-1 and SCE-4.
} 


\begin{tabular}{|c|c|}
\hline$U_{h}\left(x_{1}, T A\right)$ & 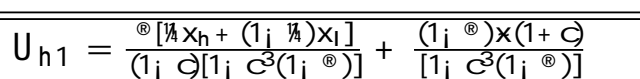 \\
\hline$U_{h}\left(\right.$ no $\left.x_{1}, T A\right)$ & 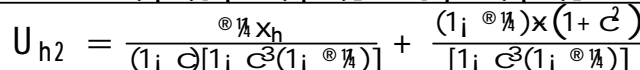 \\
\hline$U_{h}\left(x_{1}\right.$, no TA $)$ & 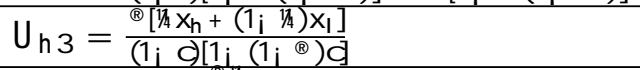 \\
\hline$U_{h}\left(\right.$ no $x_{1}$, noTA $)$ & 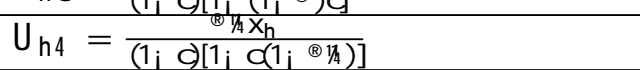 \\
\hline $\mathrm{U}_{1}\left(\mathrm{x}_{1}, \mathrm{TA}\right)$ & 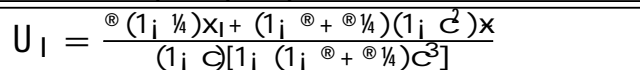 \\
\hline $\mathrm{U}_{1}\left(\right.$ no $\left.x_{1}, T A\right)$ & $\mathrm{U}_{\mathrm{ta}}=\frac{x(1+\mathrm{c})}{\left(1_{i} \mathrm{c}^{3}\right)}$ \\
\hline
\end{tabular}

where $x$ is the expected value in the TA and $c= \pm\left(1_{i}{ }^{-}\right)$.

Lemma 2 SCE-1 ( $L$ and $\mathrm{H}$ go to the TA) exists if and only if $\phi \cdot \phi_{1}^{\text {axd }}$ and $\phi \cdot \Phi \frac{\text { ad }}{2}$,

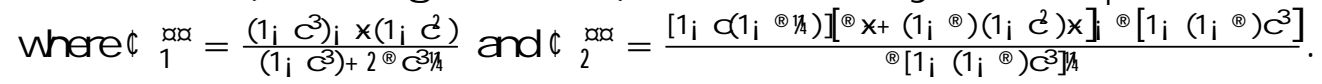

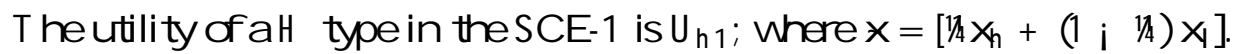

SCE-1 is an equilibrium if none possible deviation from the equilibrium is pro. table This condition requires that $\mathrm{U}_{\mathrm{h} 1}>\mathrm{U}_{\mathrm{h} 2} ; \mathrm{U}_{\mathrm{h} 3} ; \mathrm{U}_{\mathrm{h} 4}$. The low typehas no interest to deviate from the equilibrium.

2 the..r.st condition:

$$
U_{h 1}, U_{h 2}() \quad \phi \cdot \Phi_{1}^{x a d}=\frac{\left(1 ; c^{3}\right) ; \times\left(1 ; c^{2}\right)}{\left(1 ; c^{3}\right)+2 \& c^{31 / 4}}
$$

2 the second condition:

$$
U_{h 1}, U_{h 3}() \times\left(1_{i} \otimes\right)\left(c_{i} 1\right)^{2}(1+c), 0
$$

2 the third condition:

$$
\begin{aligned}
& U_{h 1}, U_{h 4}()
\end{aligned}
$$

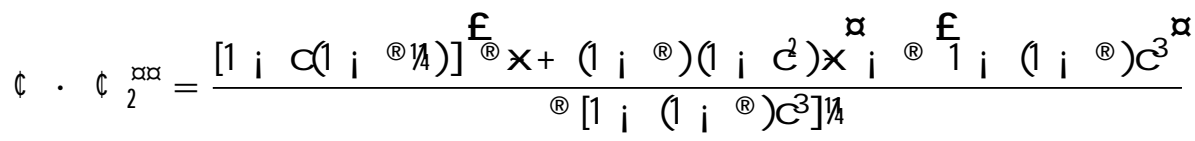

The condition (20) is always true for every parameters' value. 
Lemma 3 EE-1 ( $L$ and $H$ go to the TA) exists ix $\phi, \phi_{1}^{\infty} 13$ and $\phi \cdot \phi \frac{\text { ax }}{3}$, and $\Phi \cdot \phi_{1}^{\infty}$ where $\Phi_{3}^{\infty}=\frac{\left.x\left(1_{i} c\right)_{i}{ }^{B} c^{1 / 4} 1_{i} x\right)}{B C^{1 / 4}}$ and $\phi_{1}^{\infty}=\frac{1+c+c^{2} i x(1+c)}{1+c+c^{2}}$.

The equilibrium exist if and only if $H$ refuses to sign a contract with $L$ and wishes to go to the TA, and if and only if an $L$ type accepts another $L$ type in the search market. The utility of $H$ agent is $U_{h 2}$, where $x=\frac{\left(1_{i} 1 / 4\left(1_{i}{ }^{\circledR}+\mathbb{B}^{2} / 4 x_{1}+1 / 41_{i}\right.\right.}{1_{i}{ }^{\circledR}+2 / 4 x_{h}}$

We have an equilibrium if and only if $U_{h 2}, U_{h 1} ; U_{h 3} ; U_{h 4}$. We know by Lemma 2 that $U_{h 1}$ is al ways bigger than $U_{h 3}$ for every $x$. So we can eliminatethe second condition. The new condition will be: $\mathrm{U}_{\mathrm{h} 2}>\mathrm{U}_{\mathrm{h} 1} ; \mathrm{U}_{\mathrm{h} 4}$.

2 the ..r.st condition:

$$
U_{\mathrm{h} 2}, U_{\mathrm{h} 1}() \pitchfork, \phi_{1}^{\mathrm{ax}}
$$

2 the second condition

$$
U_{h 2}, U_{h 4}() \notin \cdot \phi_{3}^{\infty x}=\frac{x(1 ; C) ;\left(R C^{-1 / 4} 1 ; x\right)}{R C^{1 / 4}}
$$

The $L$ type has just one possible deviation: he can refuse another $L$ type and go in the TA:

$$
U_{1}, U_{t a}() \notin \cdot \phi_{1}^{x}=\frac{1+c+c^{2} i \times(1+c)}{1+c+c^{2}}
$$

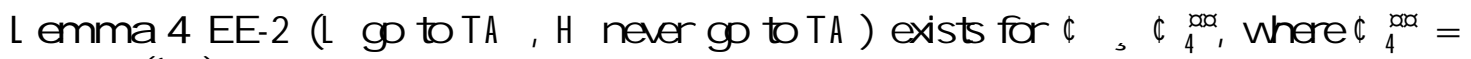
$\frac{\left(1_{\mathrm{i}} \mathrm{C}\right)}{1_{\mathrm{i} C} \mathrm{C} \mathrm{BC}^{1 / 4}}$

The utility of $\mathrm{H}$ in EE-2 is $U_{\mathrm{h} 4}$.

Equilibrium requires $U_{h 4}>U_{h 3} ; U_{h 1} ; U_{h 2}$, where $x=x_{1}$.

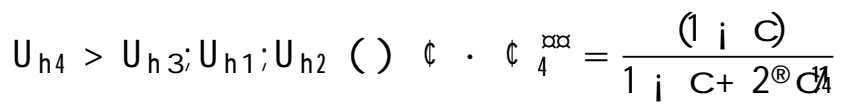

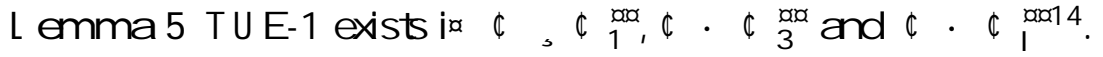

We have an equilibrium if and only if $H$ refuses to sign a contract with $L$ and wishes to go to the TA, and if and only if an $L$ type refuses another $L$ type in the search market.

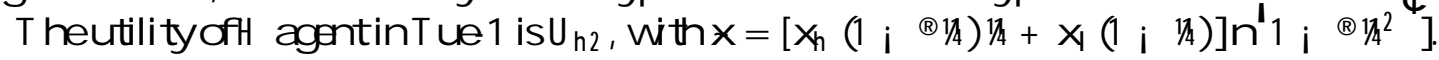
We know by Lemma 2 that $U_{h 1}$ is always bigger than $U_{h 3}$ for every $x$. So we can eliminate the second condition. The equilibrium condition is: $U_{h 2}>U_{h 1} ; U_{h 4}$.

\footnotetext{
${ }^{13}$ The value of $\phi_{1}^{\text {ax }}$ is de. ned in Lemma 2 .

${ }^{14} T$ he values $\phi_{1}^{\text {axd }}, \Phi_{3}^{\text {axd }}$ and $\phi_{1}^{\text {ax }}$ are de. ned in Lemma 2, the .r.st one, and in Lemma 3, the two others.
} 
2 the ..r.st condition:

$$
U_{h 2}, U_{h 1}() \pitchfork, \phi_{1}^{\text {ax }}
$$

2 the second condition

$$
U_{\mathrm{h} 2}, \mathrm{U}_{\mathrm{h} 4}() \pitchfork \cdot \phi_{3}^{\infty \alpha}
$$

The $L$ type has just one possible deviation: he can accepts another $L$ type in the search market. Equilibrium exist ix:

$$
U_{\text {ta }}, U_{l}() \phi, \phi_{1}^{\infty x}
$$




\section{R eferences}

[1] Bloch F. and H. Ryder (2000), "T wo-Sided Search, Marriages and Matchmakers", International Economic Review, 41 (1), 93-115.

[2] Bentolila, S. and J . J. Dolado (1994), "Labour łexibility and wages: lessons from Spain", Economic Policy, 18, 55-85.

[3] Bentolila, S. and G. Saint-Paul (1992), "The Macroeconomic Impact of Flexible Labor Contracts, With An Application to Spain", European Economic Review, 36, 1013-53.

[4] Burdett K. and M.G. Coles (1997) "Marriage and Class", Quarterly J ournal of Economics, 112, 141-168.

[5] Cahuc P. and F. Postel-Vinay (1999) "Temporary jobs, Employment Protection and Labor Market Performance", Mimeo.

[6] Mac Namara J . and E. Collins (1990) "The J ob Search Problem as an EmployerCandidate Game," J ournal of Applied Probability, 28, 815-827.

[7] Morgan P. (1994) "A model of Search, Coordination and Market segmentation,", mimeo., Department of Economics, State University of New York at Buxalo.

[8] Rioux L. (1995) “Heterogeneity, Matching, and Endogenous Labor Market Segmentation“, DELTA 95-35.

[9] Saint-Paul G. (1992) "Are the unemployed unemployable?", CEPR WP n.74.

[10] Smith L. (1993) "Cross Sectional Dynamics in a Mode of Two Sided Search", mimeo., Department of Economics, Massachusetts Institute of Technology.

[11] Wasmer, E. (1999) "Competition for J obs in a Growing Economy and the Emergence of Dualism in Employment", Economic J ournal, 109, 349-371. 